Katrin Wehrheim

\title{
Energy quantization and mean value inequalities for nonlinear boundary value problems
}

Received July 18, 2004 and in revised form October 14, 2004

\begin{abstract}
We give a unified statement and proof of a class of well known mean value inequalities for nonnegative functions with a nonlinear bound on the Laplacian. We generalize these to domains with boundary, requiring a (possibly nonlinear) bound on the normal derivative at the boundary. These inequalities give rise to an energy quantization principle for sequences of solutions of boundary value problems that have bounded energy and whose energy densities satisfy nonlinear bounds on the Laplacian and normal derivative: One obtains local uniform bounds on the complement of finitely many points, where some minimum quantum of energy concentrates.
\end{abstract}

\section{Introduction}

One purpose of this note is to explain an 'energy quantization' principle that - in different forms - has successfully been applied to a variety of partial differential equations, such as minimal submanifolds, harmonic maps, pseudoholomorphic curves, and Yang-Mills connections. The common feature of these PDE's is an energy functional. (The solutions often but not necessarily are critical points thereof.) The 'energy quantization' phenomenon which we describe in theorem 2.1 is a consequence of a mean value inequality for the energy density.

The second purpose of this note, and the content of section 3 , is a presentation and generalization of the underlying mean value inequality for the Laplace operator. Theorem 1.1 below is well known and proofs in an exhausting collection of cases can be found in the literature, e.g. [S, U]. Our aim here is to give a unified statement and proof. In theorem 1.3 we generalize this inequality to domains with boundary and inhomogeneous Neumann boundary conditions.

We denote by $B_{r}(x) \subset \mathbb{R}^{n}$ the open geodesic ball of radius $r$ centred at $x \in \mathbb{R}^{n}$ and with respect to the present metric. Integration as well as the (positive definite) Laplace operator $\Delta=\mathrm{d}^{*} \mathrm{~d}$ will also be defined with respect to the metric given in the context. The Euclidean metric on $\mathbb{R}^{n}$ is denoted by its matrix $\mathbb{1}$. Note that by our convention the Laplace operator in this metric is $\Delta=-\sum_{i=1}^{n} \partial_{i}{ }^{2}$.

K. Wehrheim: Institute for Advanced Study, Einstein Drive, Princeton, NJ 08540, USA; e-mail: wehrheim@math.princeton.edu,wehrheim@ias.edu

Mathematics Subject Classification (2000): 35B05, 35J65 
Theorem 1.1. For every $n \in \mathbb{N}$ there exist constants $C, \mu>0$, and $\delta>0$ such that the following holds for all metrics $g$ on $\mathbb{R}^{n}$ such that $\|g-\mathbb{1}\|_{W^{1, \infty}} \leq \delta$.

Let $B_{r}(0) \subset \mathbb{R}^{n}$ be the geodesic ball of radius $0<r \leq 1$. Suppose that the nonnegative function e $\in \mathcal{C}^{2}\left(B_{r}(0),[0, \infty)\right)$ satisfies for some $A_{0}, A_{1}, a \geq 0$

$$
\Delta e \leq A_{0}+A_{1} e+a e^{(n+2) / n} \quad \text { and } \quad \int_{B_{r}(0)} e \leq \mu a^{-n / 2} .
$$

Then

$$
e(0) \leq C A_{0} r^{2}+C\left(A_{1}^{n / 2}+r^{-n}\right) \int_{B_{r}(0)} e .
$$

Remark 1.2. By using local geodesic coordinates the above theorem also implies a mean value inequality on closed Riemannian manifolds with uniform constants $C, \mu>0$, and for all geodesic balls of radius less than a uniform constant.

In order to generalize the mean value inequality to manifolds with boundary we would have to consider general metrics on the half space $\mathbb{H}^{n}$. However, for the sake of an elementary geometric proof, we restrict this exposition to the Euclidean metric. We denote the intersection of a Euclidean ball with the half space by

$$
D_{r}(x):=B_{r}(x) \cap \mathbb{H}^{n}, \quad \mathbb{H}^{n}:=\left\{\left(x_{0}, \bar{x}\right) \mid x_{0} \in[0, \infty), \bar{x} \in \mathbb{R}^{n-1}\right\} .
$$

The outer unit normal derivative $\left.\frac{\partial}{\partial \nu}\right|_{\partial \mathbb{H}^{n}}$ in the Euclidean case is just $-\left.\frac{\partial}{\partial x_{0}}\right|_{x_{0}=0}$.

Theorem 1.3. For every $n \geq 2$ there exists a constant $C$ and for all $a, b \geq 0$ there exists $\mu(a, b)>0$ such that the following holds: Consider the (partial) ball $D_{r}(y) \subset \mathbb{H}^{n}$ for some $r>0$ and $y \in \mathbb{H}^{n}$. Suppose that $e \in \mathcal{C}^{2}\left(D_{r}(y),[0, \infty)\right)$ satisfies for some $A_{0}, A_{1}, B_{0}, B_{1} \geq 0$

$$
\left\{\begin{array}{cl}
\Delta e & \leq A_{0}+A_{1} e+a e^{(n+2) / n}, \\
\left.\frac{\partial}{\partial v}\right|_{\partial \mathbb{H}^{n}} e & \leq B_{0}+B_{1} e+b e^{(n+1) / n},
\end{array} \quad \text { and } \quad \int_{D_{r}(y)} e \leq \mu(a, b) .\right.
$$

Then

$$
e(y) \leq C A_{0} r^{2}+C B_{0} r+C\left(A_{1}^{n / 2}+B_{1}^{n}+r^{-n}\right) \int_{D_{r}(y)} e .
$$

\section{Energy quantization}

In this section we generally consider a PDE for maps $u: D \rightarrow T$ from a Riemannian manifold $D$ (with possibly nonempty boundary $\partial D$ ) to a target space $T$, e.g. another manifold, a Banach space, or a fibre bundle over $D$. The energy is given for all sufficiently regular maps $u$ in the form

$$
E(u)=\int_{D} e(u)
$$


where the integrand $e(u): D \rightarrow[0, \infty)$ is a nonnegative energy density function. Its key property is that for a solution $u$ of the PDE, the positive definite Laplacian $\Delta e(u)$ can be bounded above in terms of $e(u)$ itself.

If this bound is linear in $e(u)$, then theorem 1.1 provides a $\mathcal{C}^{0}$-control on $e(u)$ in terms of its mean values on geodesic balls. So if the energy $E\left(u_{i}\right)$ of a sequence of solutions $u_{i}$ is bounded, then one obtains a uniform bound on the energy densities $e\left(u_{i}\right)$ on any compact subdomain of $D \backslash \partial D$. In many cases this leads to a compactness result, i.e. to the convergence of a subsequence of the solution $u_{i}$.

For solutions of nonlinear PDE's however, the bound on $\Delta e(u)$ is usually nonlinear in $e(u)$. In that case the mean value inequality in theorem 1.1 only holds on geodesic balls with sufficiently small energy and for nonlinearities up to order $(\operatorname{dim} D+2) / \operatorname{dim} D$ in $e(u)$ (where the estimate becomes scale-invariant). Theorem 1.3 generalizes this mean value inequality to domains $D$ with boundary $\partial D$ and bounds on the outer normal derivative $\left.\frac{\partial e}{\partial v}\right|_{\partial D}$. This provides uniform bounds on $e\left(u_{i}\right)$ up to the boundary for solutions $u_{i}$ of a PDE with appropriate boundary conditions and with bounded energy.

So if a sequence of solutions $u_{i}$ has bounded energy $E\left(u_{i}\right)$ but only satisfies nonlinear bounds on the Laplacian or the normal derivative, then one only obtains locally uniform bounds on the complement of finitely many points: By a converse of the mean value inequality, the energy densities $e\left(u_{i}\right)$ can only blow up at points where some nonzero quantum of energy concentrates. In the following theorem we give a blueprint for such energy quantization results.

Here $D$ is a Riemannian manifold (possibly noncompact or with boundary), $\Delta=\mathrm{d}^{*} \mathrm{~d}$ denotes the Laplace operator, and $\frac{\partial}{\partial v}$ denotes the outer unit normal derivative at $\partial D$. For the sake of simplicity we make a technical assumption on the metric near the boundary.

Assumption: A neighbourhood of $\partial D \subset D$ is locally isometric to Euclidean $\mathbb{H}^{n}$.

For general metrics the mean value inequality at the boundary becomes more technical, but theorem 1.3 should generalize in the same way as theorem 1.1 , so this theorem should extend to general Riemannian manifolds with boundary.

Theorem 2.1. There exists a constant $\hbar>0$ depending on $n=\operatorname{dim} D$ and given constants $a, b \geq 0$ such that the following holds: Let $e_{i} \in \mathcal{C}^{2}(D,[0, \infty))$ be a sequence of nonnegative functions such that for some constants $A_{0}, A_{1}, B_{0}, B_{1} \geq 0$

$$
\left\{\begin{array}{l}
\Delta e_{i} \leq A_{0}+A_{1} e_{i}+a e_{i}^{(n+2) / n}, \\
\left.\frac{\partial}{\partial v}\right|_{\partial D} e_{i} \leq B_{0}+B_{1} e_{i}+b e_{i}^{(n+1) / n} .
\end{array}\right.
$$

Moreover, suppose that there is a uniform bound $\int_{D} e_{i} \leq E<\infty$.

Then there exist finitely many points, $x_{1}, \ldots, x_{N} \in D$ (with $N \leq E / \hbar$ ) and $a$ subsequence such that the $e_{i}$ are uniformly bounded on every compact subset of $D \backslash\left\{x_{1}, \ldots, x_{N}\right\}$, and there is a concentration of energy $\hbar>0$ at each $x_{j}$ : For every $\delta>0$ there exists $I_{j, \delta} \in \mathbb{N}$ such that

$$
\int_{B_{\delta}\left(x_{j}\right)} e_{i} \geq \hbar \quad \forall i \geq I_{j, \delta}
$$


Proof. Suppose that for some $x \in D$ there is no neighbourhood on which the $e_{i}$ are uniformly bounded. Then there exists a subsequence (again denoted $\left(e_{i}\right)$ ) and $D \ni z_{i} \rightarrow x$ such that $e_{i}\left(z_{i}\right)=R_{i}^{n}$ with $R_{i} \rightarrow \infty$. We can then apply the mean value inequality theorem 1.1 on the balls $B_{\delta_{i}}\left(z_{i}\right)$ of radius $\delta_{i}=R_{i}^{-1 / 2}>0$. For sufficiently large $i \in \mathbb{N}$, these lie within appropriate coordinate charts of $D$. In case $z \in \partial D$ we use the Euclidean coordinate charts at the boundary to apply theorem 1.3 , but we also denote the balls in half space by $B_{\delta_{i}}\left(z_{i}\right)$. Now these mean value theorems provide uniform constants $C$ and $\hbar:=\max \left\{\mu a^{-n / 2}, \mu(a, b)\right\}>0$ such that for every $i \in \mathbb{N}$ either

$$
\int_{B_{\delta_{i}}\left(z_{i}\right)} e_{i}>\hbar
$$

or $\int_{B_{\delta_{i}}\left(z_{i}\right)} e_{i} \leq \hbar$ and hence

$$
R_{i}^{n}=e\left(z_{i}\right) \leq C A_{0} \delta_{i}^{2}+C B_{0} \delta_{i}+C\left(A_{1}^{n / 2}+B_{1}^{n}+\delta_{i}^{-n}\right) \int_{B_{\delta_{i}}\left(z_{i}\right)} e_{i} .
$$

In the latter case multiplication by $\delta_{i}^{n}=R_{i}^{-n / 2}$ implies

$$
R_{i}^{n / 2} \leq C A_{0} R_{i}^{-(n+2) / 2}+C B_{0} R_{i}^{-(n+1) / 2}+C \hbar\left(A_{1}^{n / 2} R_{i}^{-n / 2}+B_{1}^{n} R_{i}^{-n / 2}+1\right) .
$$

As $i \rightarrow \infty$, the left hand side diverges to $\infty$, whereas the right hand side converges to $C \hbar$. Thus the alternative (2) must hold for all sufficiently large $i \in \mathbb{N}$. In particular, this implies the energy concentration $(1)$ at $x_{j}=x$.

Now we can go through the same argument for any other point $x \in D$ at which the present subsequence $\left(e_{i}\right)$ is not locally uniformly bounded. That way we iteratively find points $x_{j} \in D$ such that the energy concentration (1) holds for a further subsequence $\left(e_{i}\right)$. Suppose this iteration yields $N>E / \hbar$ distinct points $x_{1}, \ldots, x_{N}$ (and might not even terminate after that). Then we would have a subsequence $\left(e_{i}\right)$ for which at least energy $\hbar>0$ concentrates near each $x_{j}$. Since the points are distinct, this contradicts the energy bound $\int_{D} e_{i} \leq E$. Hence this iteration must stop after at most $\lfloor E / \hbar\rfloor$ steps, when the present subsequence $\left(e_{i}\right)$ is locally uniformly bounded in the complement of the finitely many points, where we found the energy concentration before.

The allowed nonlinearities in theorem 2.1 are sharp, and they are scale-invariant in the following sense: Consider one function $e: \mathbb{H}^{n} \rightarrow[0, \infty)$ with $\int e<\infty$ and $\Delta e \leq C e^{\lambda}$, $\frac{\partial}{\partial \nu} e \leq C e^{\mu}$ for some $\lambda, \mu \in \mathbb{R}$. Then the rescaled functions $e_{\rho}(z)=\rho^{-n} e(z / \rho)$ for $\rho>0$ have the same energy $\int e_{\rho}=\int e$ but $e_{\rho}(0)$ blows up as $\rho \rightarrow 0$. On the other hand, they satisfy the nonlinear bounds $\Delta e_{\rho} \leq C \rho^{\lambda n-n-2} e_{\rho}^{\lambda}$ and $\frac{\partial}{\partial \nu} e_{\rho} \leq C \rho^{\mu n-n-1} e_{\rho}^{\mu}$. Here the constants are bounded iff $\lambda>(n+2) / n$ and $\mu>(n+1) / n$, so the theorem cannot hold with nonlinearities of these higher orders.

The analogy in the use of energy densities in compactness proofs for a variety of PDE's, including minimal submanifolds [A, CS] and harmonic maps of surfaces [SU], has already been observed and listed by Wolfson [Wo]. Below, we will discuss pseudoholomorphic curves and Yang-Mills connections in more detail, and in the appendix we give some sample calculations for the energy densities. In local coordinates these PDE's are all second order nonlinear elliptic systems (or first order reductions thereof), whose 
leading term is a component-wise Laplacian. In all cases, the nonlinearities are exactly of the maximal order as in theorem 2.1. This corresponds to the fact that the energy functionals are conformally invariant.

For pseudoholomorphic curves (with a 2-dimensional domain) the energy is the $\mathrm{L}^{2}$ norm of the gradient, and the estimate $\Delta e \leq C\left(e+e^{2}\right)$ leads to Gromov's compactness result [G, Wo]. A detailed proof of $\Delta e \leq C e^{2}$ can be found in [MS, Lemma 4.3.1]. The linear term in the bound on $\Delta e$ only occurs when the almost complex structure varies over the domain of the pseudoholomorphic curve.

For pseudoholomorphic curves with Lagrangian boundary conditions, these compactness results are also well known. They can be proven via a specific choice of a metric for which $\frac{\partial}{\partial \nu} e=0$ (see $[\overline{\mathrm{F}}]$ and $[\overline{\mathrm{MS}}$, Lemma 4.3.3]). Then the energy density can be extended across the boundary by reflection and the mean value inequality for $\mathbb{R}^{n}$ applies. For the naturally induced metric, the Lagrangian boundary condition only implies $\frac{\partial}{\partial \nu} e \leq C\left(e+e^{3 / 2}\right)$ (see lemma A.1, which however fits nicely into our energy quantization principle.

For Yang-Mills connections on 4-manifolds the energy is the $L^{2}$-norm of the curvature. The bound $\Delta e \leq C\left(e+e^{3 / 2}\right)$ was used by Uhlenbeck [U] to prove a removable singularity result, which leads to Donaldson's compactification of the moduli space of antiself-dual instantons [D]. For a proof of the energy quantization as in theorem 2.1] see also [We, Thm. 2.1]. As an example of the calculations involved we prove the bounds on $\Delta e$ in lemma A.2 based on a Bochner-Weitzenböck formula by Bourguignon-Lawson [BL].

Consider a principal G-bundle $P \rightarrow X$ over a 4-manifold with boundary. The YangMills equation with boundary conditions for extrema $A \in \Omega^{1}\left(X, \mathfrak{g}_{P}\right)$ of the YangMills functional is the system of $\mathrm{d}_{A}^{*} F_{A}=0$ and $\left.* F_{A}\right|_{\partial X}=0$. Here $\mathfrak{g}_{P}$ is the associated bundle whose fibre is the Lie algebra of G. We show in lemma A.2 that the energy density of such Yang-Mills connections satisfies a linear bound $\frac{\partial}{\partial \nu} e \leq B e$.

The anti-self-duality equation is a first order reduction of the Yang-Mills equation for connections on 4-manifolds. The Yang-Mills boundary condition (roughly equivalent to an inhomogeneous Neumann boundary condition) turns it into an overdetermined system, similar to Neumann boundary conditions for holomorphic curves. The natural system to consider are thus anti-self-dual connections with Lagrangian boundary conditions. Locally, these are anti-self-dual connections on a product $\mathcal{U} \times P$ of a domain $\mathcal{U} \subset \mathbb{H}^{2}$ and a G-bundle $P \rightarrow \Sigma$ over a closed Riemann surface. By a Lagrangian boundary condition for a connection $A \in \Omega^{1}\left(\mathcal{U} \times \Sigma, \mathfrak{g}_{P}\right)$ we mean $\left.A\right|_{\{z\} \times \Sigma} \in \mathcal{L}$ for every $z \in \partial \mathbb{H} \cap \mathcal{U}$, where $\mathcal{L} \subset \Omega^{1}\left(\Sigma, \mathfrak{g}_{P}\right)$ is a Lagrangian (Banach) submanifold in the symplectic space of connections.

Energy quantization for this system is proven in [We, Thm. 1.2] for the SU(2)-bundle and a special class of Lagrangian submanifolds by an argument along the lines of theorem 2.1. In that case, the energy density $e: \mathcal{U} \rightarrow[0, \infty)$ is given by the slice-wise $L^{2}$-norm of the curvature, $e(A)=\int_{\Sigma}\left|F_{A}\right|^{2}$. The special Lagrangian boundary condition (which has global nature along the Riemann surface $\Sigma$ ) provides $\frac{\partial}{\partial \nu} e \leq C\left(e+e^{3 / 2}\right)$, but one only has a linear bound $\Delta e \leq g e$ with a function $g$ that cannot be bounded in terms of $e$ or a constant. However in the argument using (3) one can replace the constant $A_{1}$ by the sequence $C R_{i}^{2}$ with a constant $C$ and for sufficiently small $\hbar>0$. The 
according estimate $|g| \leq C R_{i}^{2}$ can be established (and can roughly be understood as a bound $\Delta e \leq C e^{2}$ ). This result does not follow from the standard rescaling methods for Yang-Mills connections.

\section{Mean value inequalities}

In this section we prove the mean value inequalities that were stated in the introduction and that the energy quantization principle is based on. We continue the notation of the introduction.

The special case $A_{0}=A_{1}=a=0$ of theorem 1.1 and the starting point for the proof is Morrey's $[\mathrm{M}]$ mean value inequality for subharmonic functions. A proof of the version below can be found in e.g. [LS, Thm. 2.1]. For the Euclidean metric $g=\mathbb{1}$ we give an elementary proof in lemma 3.2 below.

Proposition 3.1. For every $n \in \mathbb{N}$ there exist constants $C_{0}$ and $\delta>0$ such that the following holds for all $0<r \leq 1$ and all metrics $g$ on $\mathbb{R}^{n}$ with $\|g-\mathbb{1}\|_{W^{1, \infty}} \leq \delta$.

If $e \in \mathcal{C}^{2}\left(B_{r}(0),[0, \infty)\right)$ satisfies $\Delta e \leq 0$, then

$$
e(0) \leq C_{0} r^{-n} \int_{B_{r}(0)} e .
$$

Proof of theorem 1.1. This proof is based on the Heinz trick, which is to consider the maximum $\bar{c}$ of the function $f$ below. This allows one to replace the bound on the Laplacian by a constant depending on $\bar{c}$. One then obtains the result from the mean value inequality for subharmonic functions and a number of rearrangements in different cases.

Consider the function $f(\rho)=(1-\rho)^{n} \sup _{B_{\rho r}(0)} e$ for $\rho \in[0,1]$. It attains its maximum at some $\bar{\rho}<1$. Let $\bar{c}=\sup _{B_{\bar{\rho} r}(0)} e=e(\bar{x})$ and $\varepsilon=\frac{1}{2}(1-\bar{\rho})<\frac{1}{2}$, then

$$
e(0)=f(0) \leq f(\bar{\rho})=2^{n} \varepsilon^{n} \bar{c} .
$$

Moreover, we have for all $x \in B_{\varepsilon r}(\bar{x}) \subset B_{r}(0)$

$$
e(x) \leq \sup _{B_{(\bar{\rho}+\varepsilon) r}(0)} e=(1-\bar{\rho}-\varepsilon)^{-n} f(\bar{\rho}+\varepsilon) \leq 2^{n}(1-\bar{\rho})^{-n} f(\bar{\rho})=2^{n} \bar{c},
$$

and hence $\Delta e \leq A_{0}+2^{n} A_{1} \bar{c}+2^{n+2} a \bar{c}^{(n+2) / n}$. Now define the function

$$
v(x):=e(x)+\frac{1}{n}\left(A_{0}+2^{n} \bar{c}\left(A_{1}+4 a \bar{c}^{2 / n}\right)\right)|x-\bar{x}|^{2}
$$

with the Euclidean norm $|x-\bar{x}|$. It is nonnegative and subharmonic on $B_{\varepsilon r}(\bar{x})$ if the metric is sufficiently $\mathcal{C}^{1}$-close to $\mathbb{1}$. This is since $\Delta_{\mathbb{1}}|x-\bar{x}|^{2}=-2 n$ for the Euclidean metric and $|x-\bar{x}| \leq \varepsilon r \leq 1$ is bounded, so $\Delta|x-\bar{x}|^{2} \leq-n$ whenever $\|g-\mathbb{1}\|_{W^{1, \infty}} \leq \delta$ is sufficiently small. The control of the metric also ensures that the integral $\int_{B_{\rho r}(\bar{x})}|x-\bar{x}|^{2}$ is bounded by the following integral over the Euclidean ball $B_{2 \rho r}^{\mathbb{1}}(\bar{x})$ : With the constant $C_{1}=2^{n+3} \operatorname{Vol} S^{n-1} /(n+2)$

$$
2 \int_{B_{2 \rho r}^{\pi}(\bar{x})}|x-\bar{x}|^{2}=2 \int_{0}^{2 \rho r} t^{n+1} \operatorname{Vol} S^{n-1} \mathrm{~d} t=C_{1}(\rho r)^{n+2} .
$$


So we obtain from proposition 3.1 with $C_{2}=\max \left\{C_{0}, \frac{1}{n} 2^{n} C_{0} C_{1}\right\}$ for all $0<\rho \leq \varepsilon$

$$
\begin{aligned}
\bar{c}=v(\bar{x}) & \leq C_{0}(\rho r)^{-n} \int_{B_{\rho r}(\bar{x})} v \\
& \leq C_{2}\left(2^{-n} A_{0}+\bar{c}\left(A_{1}+4 a \bar{c}^{2 / n}\right)\right)(\rho r)^{2}+C_{2}(\rho r)^{-n} \int_{B_{\rho r}(\bar{x})} e .
\end{aligned}
$$

If $2^{-n} C_{2} A_{0}(\varepsilon r)^{2}>\frac{1}{2} \bar{c}$, then $e(0) \leq \bar{c} \leq 2^{1-n} \varepsilon^{2} C_{2} A_{0} r^{2} \leq 2^{-1-n} C_{2} A_{0} r^{2}$ proves the assertion. Otherwise we can drop $A_{0}$ from (4) while doubling the constant $C_{2}$.

Next, if $C_{2}\left(A_{1}+4 a \bar{c}^{2 / n}\right)(\rho r)^{2} \leq \frac{1}{2}$, then 44 implies $\bar{c} \leq 2 C_{2}(\rho r)^{-n} \int_{B_{r}(0)} e$. So if $C_{2}\left(A_{1}+4 a \bar{c}^{2 / n}\right)(\varepsilon r)^{2} \leq \frac{1}{2}$ then $\rho=\varepsilon$ proves the assertion,

$$
e(0) \leq 2^{n} \varepsilon^{n} \bar{c} \leq 2^{n+1} C_{2} r^{-n} \int_{B_{r}(0)} e .
$$

Otherwise we can choose $0<\rho<\varepsilon$ such that $(\rho r)^{-2}=2 C_{2}\left(A_{1}+4 a \bar{c}^{2 / n}\right)$. Then we obtain with $C_{3}=\left(2 C_{2}\right)^{1+n / 2}$

$$
e(0) \leq \bar{c} \leq C_{3}\left(A_{1}+4 a \bar{c}^{2 / n}\right)^{n / 2} \int_{B_{\rho r}(\bar{x})} e .
$$

Again we have to distinguish two cases: Firstly, if $4 a \bar{c}^{2 / n} \leq A_{1}$ then this yields

$$
e(0) \leq C_{3}\left(2 A_{1}\right)^{n / 2} \int_{B_{\rho r}(\bar{x})} e .
$$

Secondly, if $A_{1}<4 a \bar{c}^{2 / n}$ then $\bar{c}<\bar{c} C_{3}(8 a)^{n / 2} \int_{B_{\rho r}(\bar{x})} e$ and thus with $\mu=8^{-n / 2} C_{3}^{-1}>0$

$$
\int_{B_{r}(0)} e>\mu a^{-n / 2}
$$

So we either have the above or with some constant $C$ (that only depends on $n$ )

$$
e(0) \leq C A_{0} r^{2}+C\left(A_{1}^{n / 2}+r^{-n}\right) \int_{B_{r}(0)} e .
$$

Theorem 1.3 will be proven in three steps. The first step is the generalization of proposition 3.1 to domains with boundary and subharmonic functions in the sense of the weak Neumann equation: A distribution $e$ on a manifold $M$ is called subharmonic if for all $\psi \in \mathcal{C}^{\infty}(M,[0, \infty))$ with $\left.\frac{\partial \psi}{\partial v}\right|_{\partial M}=0$

$$
0 \geq \int_{M} e \Delta \psi \quad\left(=\int_{M} \psi \Delta e+\int_{\partial M} \psi \frac{\partial e}{\partial v}\right) .
$$

For $e \in \mathcal{C}^{2}(M)$ the equality above holds and implies that $\Delta e \leq 0$ and $\left.\frac{\partial e}{\partial \nu}\right|_{\partial M} \leq 0$. 
Lemma 3.2. For every $n \geq 2$ there exists a constant $C_{0}$ such that the following holds for all $R>0$ and $y \in \mathbb{H}^{n}:$ Suppose that $e \in \mathcal{C}^{2}\left(D_{r}(y),[0, \infty)\right)$ satisfies

$$
\begin{cases}\Delta e & \leq 0 \\ \left.\frac{\partial}{\partial v}\right|_{\partial \mathbb{H}^{n}} e & \leq 0\end{cases}
$$

Then

$$
e(y) \leq C_{0} r^{-n} \int_{D_{r}(y)} e .
$$

Proof. We will write $\mathbb{H}^{n}=\left\{\left(x_{0}, \bar{x}\right) \mid x_{0} \in[0, \infty), \bar{x} \in \mathbb{R}^{n-1}\right\}$ and also use spherical coordinates $\left(x_{0}, \bar{x}\right)=\left(y_{0}+r \cos \phi, \bar{y}+r \sin \phi \cdot z\right)=:(r, \phi, z)$ with $r \in[0, \infty)$, $\phi \in[0, \pi]$, and $z \in S^{n-2} \subset \mathbb{R}^{n}$. (For $n=2$ this notation means $S^{0}=\{-1,1\}$, and integration $\int_{S^{0}} \ldots \mathrm{dvol}_{S^{0}}$ will denote summation of the values at these two points.) Now the boundary of $D_{r}(y)$ has two parts,

$$
\begin{aligned}
Z_{r} & :=\partial D_{r}(y) \cap \partial \mathbb{H}^{n}=\left\{(0, \bar{x})|| \bar{x}-\left.\bar{y}\right|^{2} \leq r^{2}-y_{0}^{2}\right\}, \\
\Gamma_{r} & :=\partial B_{r}(y) \cap \mathbb{H}^{n}=\left\{(r, \phi, z) \mid \phi \in\left[0, \phi_{0}(r)\right], z \in S^{n-2}\right\} .
\end{aligned}
$$

Here we use $\phi_{0}(r):=\arccos \left(-y_{0} / r\right)$. For $y_{0}>r$ we set $\phi_{0}(r):=\pi$, so $\Gamma_{r}$ is the entire sphere and the set $Z_{r}$ is empty. With this we calculate for all $r>0$

$$
\begin{aligned}
& \frac{\mathrm{d}}{\mathrm{d} r}\left(r^{-n+1} \int_{\Gamma_{r}} e\right) \\
& =\frac{\mathrm{d}}{\mathrm{d} r}\left(r^{-n+1} \int_{0}^{\phi_{0}(r)} \int_{S^{n-2}} e(r, \phi, z)(r \sin \phi)^{n-2} \mathrm{dvol}_{S^{n-2}} r \mathrm{~d} \phi\right) \\
& =\int_{0}^{\phi_{0}(r)} \int_{S^{n-2}} \partial_{r} e(r, \phi, z)(\sin \phi)^{n-2} \mathrm{dvol}_{S^{n-2}} \mathrm{~d} \phi \\
& \quad+\frac{\partial \phi_{0}}{\partial r} \int_{S^{n-2}} e\left(r, \phi_{0}(r), z\right)\left(\sin \phi_{0}(r)\right)^{n-2} \mathrm{dvol}_{S^{n-2}} .
\end{aligned}
$$

Note that $\phi_{0}(r)$ is constant for $y_{0}=0$ as well as for $r \leq y_{0}$. So firstly in case $y_{0}>0$ we have for all $0<r \leq y_{0}$

$$
\frac{\mathrm{d}}{\mathrm{d} r}\left(r^{-n+1} \int_{\Gamma_{r}} e\right)=r^{-n+1} \int_{\partial D_{r}(y)} \frac{\partial}{\partial \nu} e=-r^{-n+1} \int_{D_{r}(y)} \Delta e \geq 0 .
$$

In that case we moreover have

$$
\lim _{r \rightarrow 0}\left(r^{-n+1} \int_{\Gamma_{r}} e\right)=\operatorname{Vol} S^{n-1} e(y)
$$

which is less than or equal to $r^{-n+1} \int_{\Gamma_{r}} e$ for all $0<r \leq y_{0}$. So integrating $\int_{0}^{R / 2} r^{n-1} \ldots \mathrm{d} r$ proves the lemma for all $R \leq 2 y_{0}$,

$$
\frac{1}{n} 2^{-n} R^{n} \operatorname{Vol} S^{n-1} e(y) \leq \int_{0}^{R / 2} \int_{\Gamma_{r}} e \mathrm{~d} r \leq \int_{D_{R}(y)} e .
$$


Next, in case $y_{0}=0$ we have for all $r>0$

$$
\begin{aligned}
\frac{\mathrm{d}}{\mathrm{d} r}\left(r^{-n+1} \int_{\Gamma_{r}} e\right) & =r^{-n+1} \int_{\Gamma_{r}} \frac{\partial}{\partial \nu} e \\
& =-r^{-n+1} \int_{D_{r}(y)} \Delta e-r^{-n+1} \int_{Z_{r}} \frac{\partial}{\partial \nu} e \geq 0 .
\end{aligned}
$$

Since $\lim _{r \rightarrow 0}\left(r^{-n+1} \int_{\Gamma_{r}} e\right)=\frac{1}{2} \operatorname{Vol} S^{n-1} e(y)$, integration over $0<r \leq R$ then proves the lemma for $y_{0}=0$ and all $R>0$,

$$
\frac{1}{2 n} R^{n} \operatorname{Vol} S^{n-1} e(y) \leq \int_{0}^{R} \int_{\Gamma_{r}} e \mathrm{~d} r=\int_{D_{R}(y)} e .
$$

Finally, in case $R>2 y_{0}>0$ we obtain from (5) for all $r>y_{0}$

$$
\frac{\mathrm{d}}{\mathrm{d} r}\left(r^{-n+1} \int_{\Gamma_{r}} e\right) \geq \frac{-y_{0}}{r \sqrt{r^{2}-y_{0}^{2}}} \int_{S^{n-2}} e\left(r, \phi_{0}(r), z\right)\left(\sin \phi_{0}(r)\right)^{n-2} \mathrm{dvol}_{S^{n-2}} .
$$

Now we can use $77, \sqrt[6]{6}$, and integrate the above to obtain for all $y_{0}<r \leq \frac{1}{2} R$

$$
\begin{aligned}
\operatorname{Vol} S^{n-1} e(y) \leq & r^{-n+1} \int_{\Gamma_{r}} e \\
& +\int_{y_{0}}^{r} y_{0} \rho^{1-n}\left(\rho^{2}-y_{0}^{2}\right)^{(n-3) / 2} \int_{S^{n-2}} e\left(\rho, \phi_{0}(\rho), z\right) \mathrm{dvol}_{S^{n-2}} \mathrm{~d} \rho .
\end{aligned}
$$

Since $\left(\rho, \phi_{0}(\rho), z\right) \in \partial \mathbb{H}^{n}$, we already know that

$$
e\left(\rho, \phi_{0}(\rho), z\right) \leq \frac{2 n}{\operatorname{Vol} S^{n-1}\left(\frac{R}{2}\right)^{n}} \int_{D_{R / 2}\left(\rho, \phi_{0}(\rho), z\right)} e \leq \frac{2^{n+1} n}{\operatorname{Vol} S^{n-1} R^{n}} \int_{D_{R}(y)} e .
$$

With this (and substituting $t=\rho / y_{0}$ ) we find that for all $0<y_{0}<r \leq \frac{1}{2} R$

$$
\begin{aligned}
\operatorname{Vol} S^{n-1} e(y) \leq & r^{-n+1} \int_{\Gamma_{r}} e \\
& +\frac{2^{n+1} n \operatorname{Vol} S^{n-2}}{R^{n} \operatorname{Vol} S^{n-1}} \int_{1}^{r y_{0}^{-1}} t^{-2}\left(1-t^{-2}\right)^{(n-3) / 2} \mathrm{~d} t \int_{D_{R}(y)} e \\
\leq & r^{-n+1} \int_{\Gamma_{r}} e+C R^{-n} \int_{D_{R}(y)} e .
\end{aligned}
$$

Here we have introduced a constant $C$ that only depends on $n \geq 2$, in particular on the value of the integral in $t$ : For $n=2$ we calculate it explicitly,

$$
\int_{1}^{r y_{0}^{-1}} t^{-2}\left(1-t^{-2}\right)^{-1 / 2} \mathrm{~d} t=\left[\arccos \left(t^{-1}\right)\right]_{1}^{r y_{0}^{-1}}=\arccos \left(\frac{r}{y_{0}}\right)<\frac{\pi}{2} .
$$


For $n \geq 3$ we have

$$
\int_{1}^{r y_{0}^{-1}} t^{-2}\left(1-t^{-2}\right)^{(n-3) / 2} \mathrm{~d} t \leq \int_{1}^{r y_{0}^{-1}} t^{-2} \mathrm{~d} t=1-\frac{r}{y_{0}}<1 .
$$

Now from (6) we know that (8) also holds for $0<r \leq y_{0}$ (with $C=0$ ), so integrating $\int_{0}^{R / 2} r^{n-1} \ldots \mathrm{d} r$ proves the lemma in this last case,

$$
\frac{1}{n}\left(\frac{R}{2}\right)^{n} \operatorname{Vol} S^{n-1} e(y) \leq \int_{0}^{R / 2} \int_{\Gamma_{r}} e \mathrm{~d} r+\frac{1}{n}\left(\frac{R}{2}\right)^{n} C R^{-n} \int_{D_{R}(y)} e \leq C_{0} \int_{D_{R}(y)} e .
$$

Proof of theorem 1.3. With lemma 3.2 in hand, the second step of the proof is to assume constant positive bounds, $\Delta e \leq A$ and $\left.\frac{\partial e}{\partial v}\right|_{\partial \mathbb{H}^{n}} \leq B$ and find a constant $C_{1}$ (only depending on $n$ ) such that for all $r>0$ and $y \in \mathbb{H}^{n}$

$$
e(y) \leq C_{1} r^{-n} \int_{D_{r}(y)} e+C_{1} A r^{2}+C_{1} B r .
$$

That is, we first prove the theorem with $A_{1}=B_{1}=a=b=0$. To do this consider the function

$$
v(x):=e(x)+\frac{1}{2 n} A|x-y|^{2}+\left(B+\frac{1}{n} A y_{0}\right) x_{0} .
$$

It is positive and satisfies $\Delta v \leq 0$ and $\left.\frac{\partial v}{\partial v}\right|_{\partial \mathbb{H}^{n}} \leq 0$, so lemma 3.2 implies that

$$
e(y)=v(y)-\left(B+\frac{1}{n} A y_{0}\right) y_{0} \leq v(y) \leq C_{0} r^{-n} \int_{D_{r}(y)} v
$$

In case $r \leq y_{0}$ we just use $v(x)=e(x)+\frac{1}{2 n} A|x-y|^{2}$, then the same holds, and moreover

$$
\int_{D_{r}(y)} v=\int_{D_{r}(y)} e+\frac{1}{2 n} A \int_{0}^{r} t^{n+1} \operatorname{Vol} S^{n-1} \mathrm{~d} t=\int_{D_{r}(y)} e+\frac{\operatorname{Vol} S^{n-1}}{2 n(n+2)} A r^{n+2} .
$$

In case $r>y_{0}$ we have (using $x_{0} \leq 2 r$ on $B_{r}(y)$ )

$$
\begin{aligned}
\int_{D_{r}(y)} v & \leq \int_{D_{r}(y)} e+\frac{1}{2 n} A \int_{0}^{r} t^{n+1} \operatorname{Vol} S^{n-1} \mathrm{~d} t+\left(B+\frac{1}{n} A y_{0}\right) \int_{D_{r}(y)} x_{0} \\
& \leq \int_{D_{r}(y)} e+\frac{\mathrm{Vol} S^{n-1}}{2 n(n+2)} A r^{n+2}+\left(B+\frac{1}{n} A r\right) \frac{2}{n} \operatorname{Vol} S^{n-1} r^{n+1}
\end{aligned}
$$

In any case, putting this into (10) proves $(9)$.

Finally, to prove the theorem we consider - analogous to the proof of theorem $1.1-$ the function $f(\rho)=(1-\rho)^{n} \sup _{D_{\rho r}(y)} e$ defined for $\rho \in[0,1]$. It attains its maximum at some $\bar{\rho}<1$. We denote $\bar{c}=\sup _{D_{\bar{\rho} r}(y)} e=e(\bar{x})$ and $\varepsilon=\frac{1}{2}(1-\bar{\rho})$, then $e(y) \leq 2^{n} \varepsilon^{n} \bar{c}$ and $e(x) \leq 2^{n} \bar{c}$ for all $x \in D_{\varepsilon r}(\bar{x})$. Thus on $D_{\varepsilon r}(\bar{x}) \subset D_{r}(y)$ we have the estimates 
$\Delta e \leq A_{0}+2^{n} \bar{c}\left(A_{1}+4 a \bar{c}^{2 / n}\right)$ and $\left.\frac{\partial e}{\partial \nu}\right|_{\partial \mathbb{H}^{n}} \leq B_{1}+2^{n} \bar{c}\left(B_{1}+2 b \bar{c}^{1 / n}\right)$. Putting this into (9) yields for all $0<\rho \leq \varepsilon$

$$
\begin{aligned}
\bar{c}=e(\bar{x}) \leq C_{1}(\rho r)^{-n} \int_{D_{\rho r}(\bar{x})} e+ & C_{1}\left(A_{0}+2^{n} \bar{c}\left(A_{1}+4 a \bar{c}^{2 / n}\right)\right)(\rho r)^{2} \\
& +C_{1}\left(B_{0}+2^{n} \bar{c}\left(B_{1}+2 b \bar{c}^{1 / n}\right)\right) \rho r .
\end{aligned}
$$

To deduce the claimed mean value inequality from this, we have to go through a number of different cases. Firstly, if $C_{1} A_{0}(\varepsilon r)^{2}+C_{1} B_{0} \varepsilon r \geq \frac{1}{2} \bar{c}$, then since $\varepsilon \leq \frac{1}{2}$

$$
e(y) \leq \bar{c} \leq C_{1} A_{0} r^{2}+C_{1} B_{0} r,
$$

which proves the theorem. Otherwise (11) continues to hold with $A_{0}$ and $B_{0}$ dropped (and the constant $C_{1}$ doubled). Next, let $0<\varepsilon^{\prime}<\varepsilon$ be the solution of the equation $A_{1}\left(\varepsilon^{\prime} r\right)^{2}+B_{1} \varepsilon^{\prime} r=2^{-n-1} C_{1}^{-1}$ or in case $A_{1}(\varepsilon r)^{2}+B_{1} \varepsilon r \leq 2^{-n-1} C_{1}^{-1}$ let $\varepsilon=\varepsilon^{\prime}$. Then we can rearrange (11) to obtain for all $0<\rho \leq \varepsilon^{\prime}$

$$
\int_{D_{r}(y)} e \geq \int_{D_{\rho r}(\bar{x})} e \geq \bar{c}(\rho r)^{n}\left(\frac{1}{2} C_{1}^{-1}-2^{n+2} a \bar{c}^{2 / n}(\rho r)^{2}-2^{n+1} b \bar{c}^{1 / n} \rho r\right) .
$$

Now if $a>0$ or $b>0$ let $\eta(a, b)>0$ be the solution of

$$
2^{n+2} a \eta^{2}+2^{n+1} b \eta=\frac{1}{4} C_{1}^{-1} .
$$

If $\bar{c}^{1 / n} \rho r=\eta(a, b)$ for some $0<\rho \leq \varepsilon^{\prime}$, then the theorem holds with

$$
\int_{D_{r}(y)} e \geq \frac{1}{4} C_{1}^{-1} \eta(a, b)^{n}=: \mu(a, b)>0 .
$$

Otherwise we must have $\bar{c}^{1 / n} \varepsilon^{\prime} r<\eta(a, b)$, so 12 with $\rho=\varepsilon^{\prime}$ gives

$$
\bar{c} \leq 4 C_{1}\left(\varepsilon^{\prime} r\right)^{-n} \int_{D_{r}(y)} e .
$$

In the special case $a=b=0$ we get the same directly from (12). In case $\varepsilon^{\prime}=\varepsilon$ this proves the theorem since $e(y) \leq 2^{n} \varepsilon^{n} \bar{c}$. Otherwise $\varepsilon^{\prime}<\varepsilon$ satisfies with $C_{2}=2^{n+1} C_{1}$

$$
\begin{aligned}
2 C_{2}^{-1} & =A_{1}\left(\varepsilon^{\prime} r\right)^{2}+B_{1} \varepsilon^{\prime} r+C_{2}^{-1} \\
& =\left(\sqrt{A_{1}} \varepsilon^{\prime} r+C_{2}^{-1 / 2}\right)^{2}+\left(B_{1}-2 C_{2}^{-1 / 2} \sqrt{A_{1}}\right) \varepsilon^{\prime} r \\
& =\left(\frac{1}{2} C_{2}^{1 / 2} B_{1} \varepsilon^{\prime} r+C_{2}^{-1 / 2}\right)^{2}+\left(A_{1}-\frac{1}{4} C_{2} B_{1}^{2}\right)\left(\varepsilon^{\prime} r\right)^{2} .
\end{aligned}
$$

From this one sees that either $B_{1} \leq 2 C_{2}^{-1 / 2} \sqrt{A_{1}}$ and $\varepsilon^{\prime} r \geq(\sqrt{2}-1) C_{2}^{-1 / 2} A_{1}^{-1 / 2}$ from the second line, or $A_{1} \leq \frac{1}{4} C_{2} B_{1}^{2}$ and $\varepsilon^{\prime} r \geq 2(\sqrt{2}-1) C_{2}^{-1} B_{1}^{-1}$ from the third line. Putting this into (13) we finally obtain in this last case with a constant $C$ that only depends on $n$

$$
e(y) \leq \bar{c} \leq C\left(A_{1}^{n / 2}+B_{1}^{n}\right) \int_{D_{r}(y)} e .
$$




\section{A. Some identities for energy densities}

Let $(M, \omega)$ be a compact symplectic manifold, let $J$ be an $\omega$-compatible almost complex structure, and fix the induced metric $\omega(\cdot, J \cdot)$ on TM. Moreover, let $L \subset M$ be a Lagrangian submanifold, that is, a submanifold of dimension $\frac{1}{2} \operatorname{dim} M$ with $\left.\omega\right|_{L} \equiv 0$.

For the following we use the coordinates $(s, t) \in \mathbb{H}^{2}$ with $t \leq 0$. Then the outer unit normal derivative on $\partial \mathbb{H}^{2}$ is $\left.\partial_{t}\right|_{t=0}$.

Lemma A.1. Let $\Omega \subset \mathbb{H}^{2}$ be an open domain and consider a pseudoholomorphic curve $u: \Omega \rightarrow M$ with Lagrangian boundary conditions,

$$
\partial_{s} u+J \partial_{t} u=0, \quad u(z) \in L \quad \forall z \in \partial \mathbb{H}^{2} \cap \Omega .
$$

Its energy density $e(u)=\left|\partial_{s} u\right|^{2}: \Omega \rightarrow[0, \infty)$ satisfies

$$
\Delta e \leq a e^{2},\left.\quad \frac{\partial}{\partial v}\right|_{\partial \mathbb{H}^{2}} e \leq b e^{3 / 2}
$$

with constants $a, b$ that only depend on $(M, \omega, J)$ and $L$.

Proof. For $\Delta e \leq a e^{2}$ see the proof of [MS, Lemma 4.3.1]. We calculate the normal derivative w.l.o.g. at $(0,0)$ in Darboux-Weinstein coordinates near $u(0,0) \in L \subset M$. So $u$ is replaced by $u=\left(u_{1}, u_{2}\right): \mathcal{U} \rightarrow \mathbb{R}^{n} \times \mathbb{R}^{n}$ on a small neighbourhood $\mathcal{U} \subset \Omega$ of $(0,0)$. In these coordinates, the symplectic structure $\omega=\omega_{0}$ is independent of $(s, t) \in \mathcal{U}$, and the Lagrangian submanifold is $\mathbb{R}^{n} \times\{0\}$, see [MS, Theorem 3.32]. However, the almost complex structure $J: \mathbb{R}^{2 n} \rightarrow \mathbb{R}^{2 n \times 2 n}$ varies with the base point. So we have $\partial_{s} u+J \partial_{t} u=0$ with $J=J \circ u: \mathcal{U} \rightarrow \mathbb{R}^{2 n \times 2 n}$, and the boundary condition becomes $u_{2}(s, 0)=0$. We can now use $\partial_{t} u=J \partial_{s} u$ to calculate

$$
\begin{aligned}
\partial_{t} e(u) & =\partial_{t}\left(\omega_{0}\left(\partial_{s} u, J \partial_{s} u\right)\right) \\
& \left.=\omega_{0}\left(\partial_{s}\left(J \partial_{s} u\right), J \partial_{s} u\right)+\omega_{0}\left(\partial_{s} u,\left(\partial_{t} J\right) \partial_{s} u\right)\right)+\omega_{0}\left(\partial_{s} u, J \partial_{s}\left(J \partial_{s} u\right)\right) \\
& =2 \omega_{0}\left(\partial_{s}^{2} u, \partial_{s} u\right)+2 \omega_{0}\left(\left(\partial_{s} J\right) \partial_{s} u, J \partial_{s} u\right)+\omega_{0}\left(\partial_{s} u,\left(\partial_{t} J\right) \partial_{s} u\right) .
\end{aligned}
$$

The first term vanishes at $(0,0)$ since $\left.\partial_{s} u_{2}\right|_{t=0}=0$ and $\left.\partial_{s}^{2} u_{2}\right|_{t=0}=0$. For the second and third term note that $\partial_{s} J=(\nabla J) \partial_{s} u$ and $\partial_{t} J=(\nabla J) J \partial_{s} u$. Hence

$$
\left.\partial_{t}\right|_{t=0} e(u) \leq 2|\nabla J| \cdot\left|\partial_{s} u\right|^{3}+|J(\nabla J) J| \cdot\left|\partial_{s} u\right|^{3} \leq B e(u)^{3 / 2},
$$

where the constant $B$ only depends on $J$ and the coordinates, so it can be chosen uniform for the compact manifold $M$.

Let $\mathrm{G}$ be a compact Lie group and denote its Lie algebra by $\mathfrak{g}$. The local trivialization of a G-bundle over a 4-manifold with boundary is $\mathrm{G} \times \mathcal{U} \rightarrow \mathcal{U}$ for some open domain $\mathcal{U} \subset \mathbb{H}^{4}$. Locally, a connection is given by a $\mathfrak{g}$-valued 1-form $A \in \Omega^{1}(\mathcal{U}, \mathfrak{g})$. It induces the exterior derivative $\mathrm{d}_{A}: \Omega^{k}(\mathcal{U}, \mathfrak{g}) \rightarrow \Omega^{k+1}(\mathcal{U}, \mathfrak{g})$ given by $\mathrm{d}_{A} \eta=\mathrm{d} \eta+\frac{1}{2}[A \wedge \eta]$, where the pairing is by the Lie bracket $[\cdot, \cdot]$ on $\mathfrak{g}$. The curvature of a connection is the 2-form $F_{A}=\mathrm{d} A+[A \wedge A]$. 
Moreover, we equip $\mathfrak{g}$ with a G-invariant metric $\langle\cdot, \cdot\rangle$ and let $\mathcal{U}$ be equipped with any Riemannian metric. The covariant derivative $\nabla_{A}$ is given for sections $\xi: \mathcal{U} \rightarrow \mathfrak{g}$ by $\nabla_{A} \xi=\mathrm{d}_{A} \xi$, but it extends to differential forms by the Leibniz rule using the Levi-Civita connection on $\mathcal{U}$. The formal dual operators of $\nabla_{A}$ and $\mathrm{d}_{A}$ are denoted by $\nabla_{A}^{*}$ and by $\mathrm{d}_{A}^{*}: \Omega^{k+1}(\mathcal{U}, \mathfrak{g}) \rightarrow \Omega^{k}(\mathcal{U}, \mathfrak{g})$

Lemma A.2. Consider a Yang-Mills connection $A \in \Omega^{1}(\mathcal{U}, \mathfrak{g})$,

$$
\mathrm{d}_{A}^{*} F_{A}=0,\left.\quad * F_{A}\right|_{\partial \mathbb{H}^{4}}=0 .
$$

Its energy density $e(A)=\left|F_{A}\right|^{2}: \mathcal{U} \rightarrow[0, \infty)$ satisfies

$$
\Delta e \leq C e+c e^{3 / 2},\left.\quad \frac{\partial}{\partial v}\right|_{\partial \mathbb{H}^{4}} e \leq B e
$$

with constants $B, c, C$ that only depend on the metric on $\mathcal{U}$.

Proof. The Bochner-Weitzenböck formula [BL, Thm. 3.10] is

$$
\left(\mathrm{d}_{A} \mathrm{~d}_{A}^{*}+\mathrm{d}_{A}^{*} \mathrm{~d}_{A}\right) F_{A}=\nabla_{A}^{*} \nabla_{A} F_{A}+F_{A} \circ(\operatorname{Ric} \wedge g+2 R)+\mathcal{R}^{A}\left(F_{A}\right) .
$$

The left hand side vanishes for a Yang-Mills connection due to $\mathrm{d}_{A}^{*} F_{A}=0$ and the Bianchi identity $\mathrm{d}_{A} F_{A}=0$. The quadratic term $\mathcal{R}^{A}\left(F_{A}\right) \in \Omega^{2}(\mathcal{U}, \mathfrak{g})$ can be expressed with the help of a local orthonormal frame $\left(e_{1}, \ldots, e_{4}\right)$ of TU as

$$
\mathcal{R}^{A}\left(F_{A}\right)(X, Y)=2 \sum_{j=1}^{4}\left[F_{A}\left(e_{j}, X\right), F_{A}\left(e_{j}, Y\right)\right] .
$$

The estimate for the Laplacian now follows from

$$
\begin{aligned}
-\nabla^{*} \nabla\left|F_{A}\right|^{2} & =-2\left|\nabla_{A} F_{A}\right|^{2}-2\left\langle F_{A}, \nabla_{A}^{*} \nabla_{A} F_{A}\right\rangle \\
& \leq 2\left\langle F_{A}, F_{A} \circ(\operatorname{Ric} \wedge g+2 R)\right\rangle+2\left\langle F_{A}, \mathcal{R}^{A}\left(F_{A}\right)\right\rangle \\
& \leq C\left|F_{A}\right|^{2}+c\left|F_{A}\right|^{3} .
\end{aligned}
$$

Here the constant $C$ depends on the Ricci transform Ric and the scalar curvature $R$ of the metric on $\mathcal{U}$. The constant $c$ only depends on the metric on $\mathfrak{g}$.

For the normal derivative at w.l.o.g. 0 we use local geodesic coordinates on $\partial \mathbb{H}^{4}$ combined with the flow of a unit normal vector field into $\mathbb{H}^{4}$ to obtain coordinates $\left(x_{0}, x_{1}, x_{2}, x_{3}\right), x_{0} \leq 0$ on a neighbourhood $\mathcal{U} \subset \mathbb{H}^{4}$ of 0 . Then the components of the metric satisfy $\left.g_{i j}\right|_{x_{0}=0}=\delta_{i j}$ and we have $\left.\frac{\partial}{\partial v}\right|_{\partial \mathbb{H}^{4}}=\left.\partial_{0}\right|_{x_{0}=0}$. The boundary condition becomes $F_{0 i}=0$ for all $i$, and it implies $\nabla_{j} F_{0 i}=0$ for all $i, j \neq 0$. (Here $F_{i j}$ and $\nabla_{j}$ denote the components of $F_{A}$ and $\nabla_{A}$.) With this we can calculate

$$
\begin{aligned}
\left.\partial_{0}\right|_{x_{0}=0}\left|F_{A}\right|^{2} & =\left.\partial_{0}\right|_{x_{0}=0} \sum_{i, j, k, \ell} g^{i j} g^{k \ell}\left\langle F_{i k}, F_{j \ell}\right\rangle \\
& =2 \sum_{i, j, k} \partial_{0} g^{i j}\left\langle F_{i k}, F_{j k}\right\rangle+2 \sum_{i, k}\left\langle\nabla_{0} F_{i k}, F_{i k}\right\rangle \leq B\left|F_{A}\right|^{2} .
\end{aligned}
$$


Here the constant $B$ only depends on the first normal derivative of the metric. The second term vanishes since $F_{i k}=0$ unless $i, k \neq 0$, but by the Bianchi identity and the boundary condition

$$
\nabla_{0} F_{i k}=\nabla_{i} F_{0 k}+\nabla_{k} F_{i 0}=0 \quad \forall i, k \neq 0
$$

Acknowledgements. I am very grateful to Dietmar Salamon, Kai Cieliebak, and the referee for many helpful comments. I would also like to thank the participants and organizers of the 2004 Program for Women in Mathematics at the IAS in Princeton for providing a stimulating atmosphere and test audience.

\section{References}

[A] Anderson, M.: Local estimates for minimal submanifolds in dimensions greater than two. In: Proc. Sympos. Pure Math. 44, Amer. Math. Soc., 131-137 (1986) Zbl 0604.53022 MR 0840269

[BL] Bourguignon, J.-P., Lawson Jr., H. B.: Stability and isolation phenomena for Yang-Mills fields. Comm. Math. Phys. 79, 189-230 (1981) Zbl 0475.53060 MR 0612248

[CS] Choi, H. I., Schoen, R.: The space of minimal embeddings of a surface into a threedimensional manifold of positive Ricci curvature. Invent. Math. 81, 387-394 (1985) Zbl 0577.53044 MR 0807063

[D] Donaldson, S. K.: Polynomial invariants of smooth four-manifolds. Topology 29, 257-315 (1990) Zbl 0715.57007 MR 1066174

[F] Frauenfelder, U.: Gromov convergence of pseudoholomorphic discs. Diploma thesis, ETH Zürich, 2000

[G] Gromov, M.: Pseudoholomorphic curves in symplectic manifolds. Invent. Math. 82, 307-347 (1985) Zbl 0592.53025 MR 0809718

[LS] Li, P., Schoen, R.: $L^{p}$ and mean value properties of subharmonic functions on Riemannian manifolds. Acta Math. 153, 279-301 (1984) Zbl 0556.31005 MR 0766266

[MS] McDuff, D., Salamon, D.: J-Holomorphic Curves and Symplectic Topology. Amer. Math. Soc. Colloq. Publ. 52, Amer. Math. Soc. (2004) Zbl pre02121182 MR 2045629

[M] Morrey, C. B.: Multiple Integrals in the Calculus of Variations. Springer, New York (1966) Zbl 0142.38701 MR 0202511

[SU] Sacks, J., Uhlenbeck, K.: The existence of minimal immersions of 2-spheres. Ann. of Math. 113, 1-24 (1981) Zbl 0462.58014 MR 0604040

[S] Salamon, D.: Morse theory, the Conley index and Floer homology. Bull. London Math. Soc. 22, 113-140 (1990) Zbl 0709.58011 MR 1045282

[U] Uhlenbeck, K.: Removable singularities in Yang-Mills fields. Comm. Math. Phys. 83, 11-29 (1982) Zbl 0491.58032 MR 0648355

[We] Wehrheim, K.: Anti-self-dual instantons with Lagrangian boundary conditions II: Bubbling. Comm. Math. Phys., to appear

[Wo] Wolfson, J. G.: Gromov's compactness of pseudo-holomorphic curves and symplectic geometry. J. Differential Geom. 28, 383-405 (1988) Zbl 0661.53024 MR 0965221 